\title{
An architecture analysis approach for supporting black-box software development.
}

\begin{abstract}
A typical component-based system architecture comprises a set of components that have been purposefully designed and structured to ensure that they have "pluggable" interfaces and an acceptable match with a defined system context. However, the black-box nature of many software components means there is never a clean match between system specifications and concrete software components. Systematic architecture analysis can provide an effective, rapid and relatively low-cost mechanism for addressing risks resulting from architectural adaptation and trade-offs. However, a review of current architecture analysis approaches reveals they differ widely with respect to their ability to support black-box software development. This paper describes an analysis approach that integrates the strengths of current approaches to provide a practical architecture analysis framework for black-box component-based development. The approach is illustrated using a real case study.
\end{abstract}

Keyword: Architectural analysis; Components; Services; Black-box. 